\title{
Using Assistive Robotic Technology in Motor Neurorehabilitation After Childhood Stroke
}

\author{
Duncan L Turner ${ }^{\mathrm{a}, \mathrm{b}, \mathrm{c}, \mathrm{d}}$, William Winterbotham ${ }^{\mathrm{a}}$, Miriam Kmetova ${ }^{\mathrm{a}}$
}

\begin{abstract}
An intracranial haemorrhage resulted in severe motor impairment in the right upper limb in a previously physically-active young adolescent. Robot-assisted therapy was implemented over the course of 9 months to reduce motor impairment in the affected right upper limb. Robot-assisted therapy consisted of twice weekly sessions containing repetitive and progressively intense practice of hand grasping and arm reaching. The amount of assistance offered by the robot during grasping and reaching was adaptive such that as impairment reduced, the robot offered less assistance. Motor impairment was evaluated with the Fugl-Meyer assessment score for the upper limb. The robot-assisted therapy was associated with an increase in a clinical outcome measure of motor impairment following childhood stroke. The increase was distributed across both upper and lower arm segments. The final, stable reduction in motor impairment in the patient matched that demonstrated in a group of
\end{abstract}

Manuscript accepted for publication April 18, 2012

\footnotetext{
${ }^{a}$ Lewin Stroke Rehabilitation Unit, Cambridge University Hospital NHS Foundation Trust, Addenbrooke's Hospital, Hills Road, Cambridge CB2 $0 Q Q$, United Kingdom

${ }^{b}$ Department of Clinical Neurosciences, Cambridge University Hospital NHS Foundation Trust, Addenbrooke's Hospital, Hills Road, Cambridge CB2 0QQ, United Kingdom

${ }^{\mathrm{C}}$ Neurorehabilitation Unit, School of Health, Sport and Bioscience, University of East London, Stratford E15 4LZ, United Kingdom

${ }^{\mathrm{d}}$ Corresponding author: Duncan L Turner, School of Health, Sport and Bioscience, University of East London, Water Lane, Stratford E15 4LZ, United Kingdom. Email: d.1.turner@uel.ac.uk
}

doi:10.4021/jnr98w adult unilateral stroke survivors receiving similar treatment with robotics early in their recovery process. We conclude that robotassisted therapy offers a promising treatment option in childhood stroke involving severe upper limb motor impairment. Robot-assisted therapy could be implemented safely in an acute in-patient hospital unit and be continued after discharge to the community setting.

Keywords: Intracranial haemorrhage; Robot-aided therapy; Upper limb

\section{Introduction}

Stroke is uncommon in children, but is an important cause of mortality and morbidity with two-thirds having persistent neurological deficits, learning or developmental problems $[1,2]$. Ischaemic and heamorrhagic strokes occur in equal proportions in children compared to the larger preponderance of ischaemic stroke in adults $(\sim 85 \%)$. Reduction in quality of life, motor function impairment, visuospatial and cognitive deficits and lowered social function can remain beyond one year and up to five years after childhood stroke [1, $3]$. As a consequence, the one and five year total direct costs associated with all-cause childhood strokes are as high as for adult stroke care and more so following haemorrhagic compared to ischaemic childhood stroke (i.e. $\sim \$ 150,000$ ) [4]. The cost of intensive motor rehabilitation services within the total "other/nursing" health-related costs is unknown for childhood stroke [5]. However, a recent trial in adult chronic stroke survivors suggested that robot-assisted therapy was associated with similar total health costs to usual care or intensity matched physical therapy over the course of 9 months recovery and thus might offer a treatment option for childhood stroke as well $[6,7]$.

Therefore for the first time in this case report, robotassisted therapy was incorporated into usual care to demonstrate treatment safety and feasibility following childhood stroke and specifically to reduce the initial severe motor impairment. Robot-assisted therapy was introduced within the first 3 months as early treatment elicits greater reductions in 


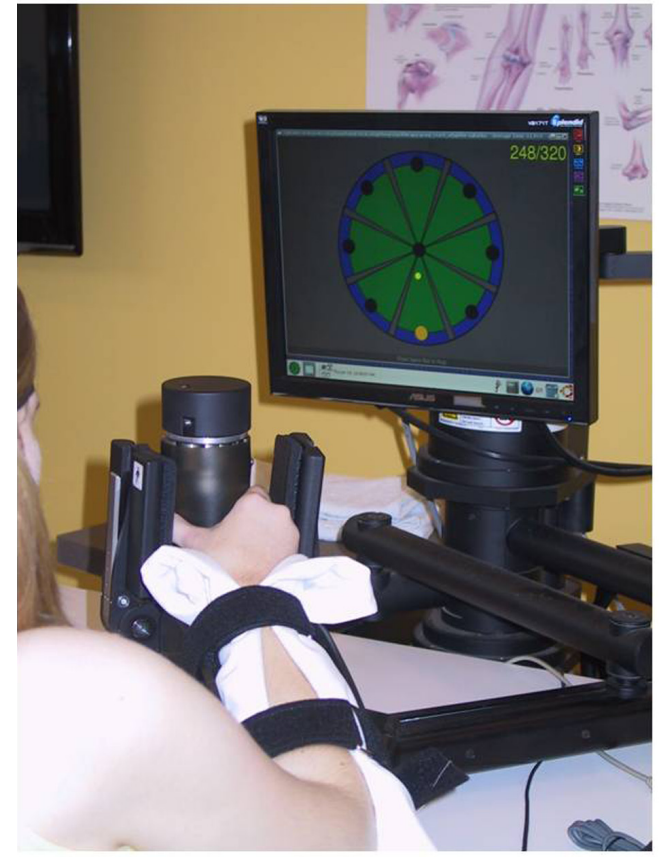

Figure 1. Robot-assisted therapy setup. The patient's lower arm is supported in a trough and the hand placed around a joystick. The visual screen illustrates the reach-grasp-reachrelease assisted therapy game, whereby repeated movements are made to 8 peripheral targets and back again to the central hold position. Both reaching of the shoulder/upper arm and the extension/flexion of the fingers and thumb are assisted and synchronised, such that the robot devices enable successful tasks to be completed even if the patient cannot move at all, for example at the beginning of the treatment programme.

motor impairment compared to its use in the chronic stage in adults (> 6 months post-stroke) $[6,8-10]$.

\section{Case Report}

\section{Patient history}

A 17 years old right-handed female collapsed whilst on holiday. Acute neurological assessment suggested an intracranial haemorrhage of idiopathic origin which was confirmed 4 months later using MRI imaging. There was low T2 signal intensity haemosiderin staining in the left cerebral peduncle, left midbrain tegmentum and left superior and middle cerebellar peduncles following the haemorrhage.

Symptoms included severe right-sided weakness and motor impairment, left-sided ataxia and left-sided visual problems. There was no motor weakness on the left-side. The symptoms and neurological findings were consistent with a brainstem/cortical-spinal/cerebellar pattern characterized by coexistence of ataxia on one body side and pyramidal disorders on the other (right-side in this patient).

\section{Experimental design}

The robot-assisted therapy programme was designed by the attending therapists and began once the patient had reached a medically stable stage of recovery at 11 weeks after the stroke episode. Robot-assisted therapy was employed to facilitate a reduction in motor impairment to a stable and maintained level in the affected right upper limb. The protocol was in accordance with the Declaration of Helsinki and ethical standards of the regional committee on human experimentation.

\section{Robot-assisted therapy}

At 100 days post-stroke, the patient began robot-assisted therapy for the motor impaired right upper limb. Two, onehour therapy sessions were undertaken per week. Each therapy session consisted of 80 robot-assisted right-sided hand grasp extension/flexions and 160 - 240 robot-assisted rightsided reach-grasp-reach-releases using a shoulder/upper arm robot device fitted with a hand module (models IMT2 plus IMT5; InMotion Technologies Inc., Boston, MA, USA; Fig. 1) $[6,9]$. The robot-assisted therapy finished at 299 days post-stroke and 59 sessions were completed.

\section{Evaluation of motor impairment}

The primary outcome measure for assessing motor impairment was the Fugl-Meyer score for the upper limb (total F-M score out of 66) [11]. This outcome measure was used during therapy to track changes in impairment with time and the number of robot sessions undertaken. The evaluations were performed by an experienced therapist following a standardised protocol adapted for robot-assisted therapy in acute and chronic adult stroke patients [6,9]. Follow-up measures of motor impairment were taken 4 weeks after the end of robot-assisted therapy and compared to similar follow-up measures in adult stroke patients in early recovery [9].

\section{Results}

Right arm total F-M score increased over the course of robot-assisted therapy. The rate of increase was greatest over the first 25 sessions ( 3 months; Fig. 2A). Robot-assisted therapy using shoulder/upper arm and hand devices was associated with improvements of both upper arm and lower hand/ wrist F-M subsection scores (Fig. 2A). The increase in total F-M score was slower (in terms of time) in the childhood stroke patient compared to a group of adult stroke patients early in robot-assisted recovery, but did eventually reach a similar stable level of motor impairment at the end of robotassisted therapy and at one month follow-up (Fig. 2B) [9]. However, the rate of development of total F-M score dur- 

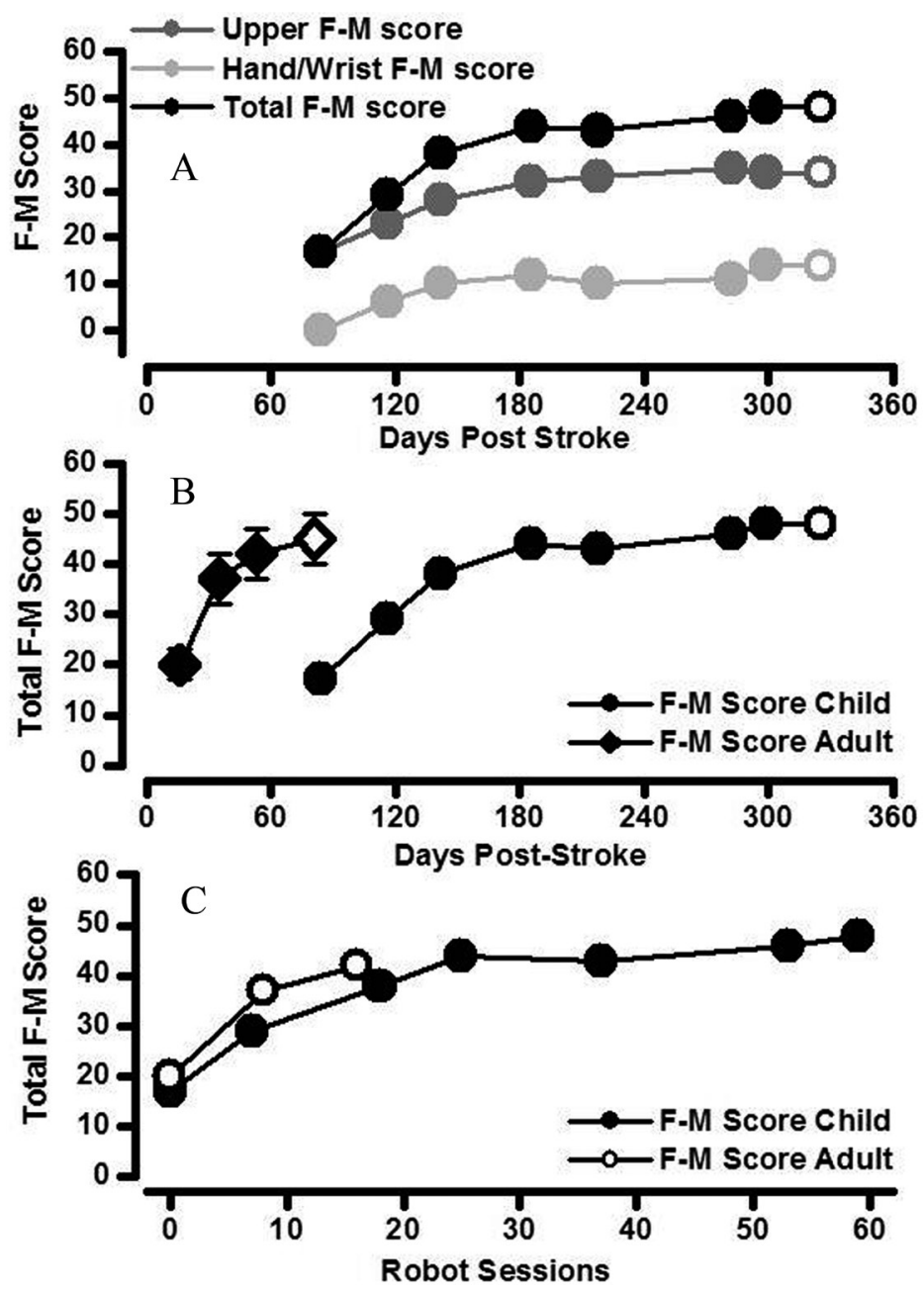

Figure 2. Changes in clinically assessed motor impairment. A. The increase in total arm F-M score (black circles) was the result of additive increases in both upper arm F-M score (score out of 42; dark grey) and hand/wrist F-M score (score out of 24; light grey) in the child stroke patient. Increases in arm segment F-M scores were maintained at one month follow-up in the child stroke patient (open symbols). B. Total F-M score for right upper limb of the childhood stroke patient (closed circles) increased relatively more quickly with time since stroke in the first half of the robot-assisted therapy (i.e. over first 3 months). The rate of increase in total F-M score with time since stroke for the child stroke patient was slower than that of a cohort of adult acute stroke patients (closed diamonds are mean \pm standard error bars; $N=10$ ) [9]. Increases in total F-M score were maintained at one month follow-up in both child and adult stroke patients (open symbols). C. The change in total F-M score was similar in child and adult stroke patients when compared as a function of robot-assisted therapy session number.

ing robot-assisted therapy was similar in adult and childhood patients when expressed in terms of the number of robot sessions (Fig. 2C). The treatment schedule was maintained throughout the rehabilitation period with no adverse effects or events.

\section{Discussion}

This case report demonstrated that robot-assisted therapy can safely be incorporated into recovery from childhood stroke. Motor impairment was comparable at the beginning of treat- ment in the childhood stroke patient to a recent comparator adult stroke group [9]. Motor impairment was reduced to a similar level during, and maintained at follow-up after, robotassisted therapy when compared to the adult acute stroke group.

Robot-assisted therapy has been shown to significantly improve motor impairment when administered early after adult stroke compared to usual care [8]. In a recent random controlled trial, robot-assisted therapy also resulted in significant improvement in motor impairment in adult chronic stroke survivors [6]. The improvement of motor impairment in chronic patients was more modest than in (sub)acute patients suggesting early incorporation of robot-assisted thera- 
py might be more beneficial as employed in this case report $[6,10]$.

Thusfar, the use of robot-assisted therapy for treating motor impairment of the upper limb in children has been restricted mainly to cerebral palsy and some years after perinatal brain injury [12]. The authors are not aware of early use of robot-assisted upper limb therapy for neurological conditions in childhood. However, the present case study suggests that robot-assisted therapy can facilitate a similar robot-session number-dependent change in motor impairment when compared to early robot-assisted therapy in adult stroke [9].

The robot assistive technology used in this case study can enable repetitive, intensive and progressive movement of parts of the upper limb [10]. The mechanical design allows adaptive interaction between robot and patient. Thus when the patient could not move the upper limb because of severe impairment (i.e. a total F-M score of 18 at the beginning of robot-assisted therapy in this patient), the robot guides the arm through a reaching or grasping task. As motor impairment reduces, the robot device offered less assistance, thereby making the motor rehabilitation progressive. The robot-assisted therapy protocol consisted of 2 one hour sessions per week and was based on that used previously in trials $[6,8-10]$.

\section{Implications and limitations}

The current case report was carried out in a publicly funded hospital/community healthcare system and financially supported by a "complex condition" tariff arrangement. The cost-effectiveness of robot-assisted therapy was recently shown to be equivalent to intensive physical therapy and importantly to long term usual care with the consequent follow-up health-related costs in chronic adult stroke survivors [7]. The social and economic impact of stroke is increasing in the working age population and the total cost of stroke to the European community is currently 64B Euros per year [13]. Thus the use of robot-assisted therapy may be an attractive treatment.

Whilst encouraging, this case report needs to be repeated in a larger childhood stroke cohort containing both ischaemic and haemorrhagic patients in order to assess differential treatment costs, effectiveness and improvements in quality of life before fuller implementation in clinical practice.

\section{Conflicting Interests}

None.

\section{References}

1. Lo W, Zamel K, Ponnappa K, Allen A, Chisolm D, Tang $\mathrm{M}$, Kerlin B, et al. The cost of pediatric stroke care and rehabilitation. Stroke. 2008;39(1):161-165.

2. Tsze DS, Valente JH. Pediatric stroke: A review. Emergency Medicine International. Epub ahead of print 2011. DOI: $10.1155 / 2011 / 734506$.

3. Everts R, Pavlovic J, Kaufmann F, Uhlenberg B, Seidel U, Nedeltchev K, Perrig W, et al. Cognitive functioning, behavior, and quality of life after stroke in childhood. Child Neuropsychol. 2008;14(4):323-338.

4. Gardner MA, Hills NK, Sidney S, Johnston SC, Fullerton HJ. The 5-year direct medical cost of neonatal and childhood stroke in a population-based cohort. Neurology. 2010;74(5):372-378.

5. Turney CM, Wang W, Seiber E, Lo W. Acute pediatric stroke: contributors to institutional cost. Stroke. 2011;42(11):3219-3225.

6. Lo AC, Guarino PD, Richards LG, Haselkorn JK, Wittenberg GF, Federman DG, Ringer RJ, et al. Robotassisted therapy for long-term upper-limb impairment after stroke. N Engl J Med. 2010;362(19):1772-1783.

7. Wagner TH, Lo AC, Peduzzi P, Bravata DM, Huang GD, Krebs HI, Ringer RJ, et al. An economic analysis of robot-assisted therapy for long-term upper-limb impairment after stroke. Stroke. 2011;42(9):2630-2632.

8. Masiero S, Celia A, Rosati G, Armani M. Robotic-assisted rehabilitation of the upper limb after acute stroke. Arch Phys Med Rehabil. 2007;88(2):142-149.

9. Turner DL, Tang X, Winterbotham W, Kmetova M. Recovery of submaximal upper limb force production is correlated with better arm position control and motor impairment early after a stroke. Clin Neurophysiol. 2012;123(1):183-192.

10. Volpe BT, Huerta PT, Zipse JL, Rykman A, Edwards D, Dipietro L, Hogan N, et al. Robotic devices as therapeutic and diagnostic tools for stroke recovery. Arch Neurol. 2009;66(9):1086-1090.

11. Fugl-Meyer AR, Jaasko L, Leyman I, Olsson S, Steglind S. The post-stroke hemiplegic patient. 1. a method for evaluation of physical performance. Scand J Rehabil Med. 1975;7(1):13-31.

12. Fasoli SE, Fragala-Pinkham M, Hughes R, Hogan N, Krebs HI, Stein J. Upper limb robotic therapy for children with hemiplegia. Am J Phys Med Rehabil. 2008;87(11):929-936.

13. Olesen J, Gustavsson A, Svensson M, Wittchen HU, Jonsson B. The economic cost of brain disorders in Europe. Eur J Neurol. 2012;19(1):155-162. 